\section{Examination of Existent Propagation Models Over Large Inhomogeneous Terrain Profiles Using Fast Integral Equation Solution}

\author{
Celal Alp Tunc, Ayhan Altintas, and Vakur B. Ertürk
}

\begin{abstract}
The accuracy of most widely used empirical models are investigated using the spectrally accelerated forward-backward (FBSA) method as a benchmark solution. First, FBSA results are obtained for propagation over large scale terrain profiles and compared with measurements to assess the accuracy of FBSA. Then, accuracy of some International Telecommunication Union (ITU) and Federal Communications Commission (FCC) propagation models are investigated. It has been observed that, for rural areas, the prediction of the most recent ITU recommended propagation model (Rec. 1546) deviates much more than older models do.
\end{abstract}

Index Terms-Federal Communications Commission (FCC) curves, forward-backward spectral acceleration (FBSA) method, International Telecommunication Union (ITU) recommendations, propagation models, rough surface scattering.

\section{INTRODUCTION}

Most of the automated propagation prediction tools for coverage analysis over geometrical databases use empirical models [1]-[5] with or without semi-empirical multiple knife-edge diffraction (MD) losses [6]-[10] in order to predict field strengths over terrain profiles. These empirical models which are described by equations or curves derived from statistical analysis of a large number of measured data, are simple and do not require details of the terrain. Therefore, they are easy and fast to apply. However, they cannot provide a very accurate estimation of the scattered field or the path loss for an arbitrary environment. Hence, comparison of empirical models in terms of accuracy is an important issue for the prediction of field strengths over large terrain profiles.

In this paper, a detailed investigation of some of the most widely used empirical propagation models with or without MD corrections has been performed using the spectrally accelerated forward-backward (FBSA) method [11]-[13] as a benchmark solution, after its accuracy is compared with measurements. Furthermore, the good agreement between the FBSA and measured results confirm the consistency of the method to be used for a section of the three-dimensional (3-D) environment, though the FBSA is based on the two-dimensional (2-D) Green's function. Use of other 2-D Green's function based integral equations for 3-D environments has been presented in the literature before [14]-[21]. We have chosen the FBSA among these methods, because of its $O(N)$ computational cost, to examine the propagation models over electrically large terrain profiles.

Interestingly, it has been observed that, for rural areas, the most recent International Telecommunication Union (ITU) recommended propagation model (Rec.-1546) needs to be modified. Furthermore, the use of MD losses in conjunction with empirical solutions increases the error if the field strength or the path loss due to the empirical model is already lower than that of the reference solution. Therefore, results of this study may help in the choice of the most suitable empirical models or in the development of more robust propagation techniques. A robust technique for the prediction of field strengths over large terrain profiles must be polarization and frequency dependent, and must take electrical properties, and details of the terrain profile into account.

Manuscript received November 12, 2004; revised March 10, 2005.

The authors are with the Department of Electrical and Electronics Engineering, Bilkent University, Ankara 06800, Turkey (e-mail: celal@ee.bilkent.edu.tr).

Digital Object Identifier 10.1109/TAP.2005.854548

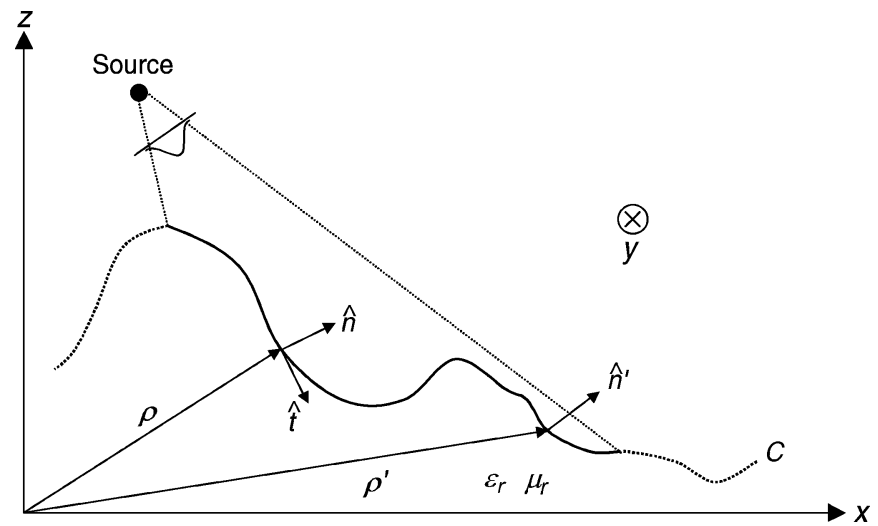

Fig. 1. Generic terrain profile.

In Section II, the integral equation (IE) formulation and its solution using the FBSA is briefly discussed. Numerical results are presented in Section III where the accuracy of existing empirical propagation models are compared. Finally, some concluding remarks are presented. An $e^{j w t}$ time convention is used and suppressed from the expressions.

\section{FORMULATION}

The scattered field over an electrically large rough terrain profile which is illuminated by an incident electromagnetic field $\left\{\mathbf{E}^{\text {inc }}(\boldsymbol{\rho})\right.$, $\left.\mathbf{H}^{\text {inc }}(\boldsymbol{\rho})\right\}(\boldsymbol{\rho}=\hat{x} x+\hat{z} z)$ is computed using an IE based method to be used as a reference solution. Fig. 1 illustrates such a rough surface that is characterized with the curve $C$ defined by $z=f(x)$, along the $x$-axis. Considering the terrain as an imperfect conductor $\left(\epsilon_{r}(\boldsymbol{\rho}), \mu_{r}(\boldsymbol{\rho})\right)$ and using the Impedance boundary conditions (IBC) [22], an electric field integral equation (EFIE) for a transverse magnetic $\left(\mathrm{TM}_{y}\right)$ polarization can be written in terms of the equivalent electric current density $J_{y}$ on the surface as

$$
\begin{aligned}
-E_{y}^{\text {inc }}(\boldsymbol{\rho})=-\eta_{s}(\boldsymbol{\rho}) J_{y}(\boldsymbol{\rho}) & -j \omega \mu \int_{C} J_{y}\left(\boldsymbol{\rho}^{\prime}\right) G\left(\boldsymbol{\rho}, \boldsymbol{\rho}^{\prime}\right) d \rho^{\prime} \\
& +\int_{C} \eta_{s}\left(\boldsymbol{\rho}^{\prime}\right) J_{y}\left(\boldsymbol{\rho}^{\prime}\right) \frac{\partial}{\partial n^{\prime}} G\left(\boldsymbol{\rho}, \boldsymbol{\rho}^{\prime}\right) d \rho^{\prime}
\end{aligned}
$$

whereas a magnetic field integral equation (MFIE) for the transverse electric $\left(\mathrm{TE}_{y}\right)$ polarization case can be obtained in terms of the tangential induced current $J_{t}$ as

$$
\begin{aligned}
-H_{y}^{\text {inc }}(\boldsymbol{\rho})=J_{t}(\boldsymbol{\rho})-\int_{C} J_{t}\left(\boldsymbol{\rho}^{\prime}\right) \frac{\partial}{\partial n^{\prime}} G\left(\boldsymbol{\rho}, \boldsymbol{\rho}^{\prime}\right) d \rho^{\prime} \\
\\
\quad+j \omega \epsilon \int_{C} \eta_{s}\left(\boldsymbol{\rho}^{\prime}\right) J_{t}\left(\boldsymbol{\rho}^{\prime}\right) G\left(\boldsymbol{\rho}, \boldsymbol{\rho}^{\prime}\right) d \rho^{\prime} .
\end{aligned}
$$

In (1) and (2), $\eta_{s}$ is the surface impedance along the surface, $G\left(\boldsymbol{\rho}, \boldsymbol{\rho}^{\prime}\right)=H_{0}^{(2)}\left(\boldsymbol{\rho}, \boldsymbol{\rho}^{\prime}\right) / 4 j$ is the 2-D Green's function and $\left(\partial / \partial n^{\prime}\right) G\left(\boldsymbol{\rho}, \boldsymbol{\rho}^{\prime}\right)$ denotes its derivative with respect to $\hat{n}^{\prime}$, the normal vector to the surface at the radiating point $\boldsymbol{\rho}^{\prime}$. It is noted that $\mathrm{TM}_{y}$ and $\mathrm{TE}_{y}$ definitions used here are with respect to $y$-coordinate.

Assuming that the incident field is of finite extent in space, the surface and integrals in (1) and (2) can be confined to a finite region, though the profile $C$ is arbitrarily extended to infinity. Therefore, (1) and (2) can be solved using a point-matching moment method solution leading to the matrix equation in the form of

$$
\mathbf{V}=\overline{\mathbf{Z}} \cdot \mathbf{I}
$$

where I contains the unknown current coefficients $I_{m}, \overline{\mathbf{Z}}$ is the impedance matrix whose entries are given in [13], and $\mathbf{V}$ denotes the incident field evaluated at the matching points. 
(a) Hadsund Terrain Profile

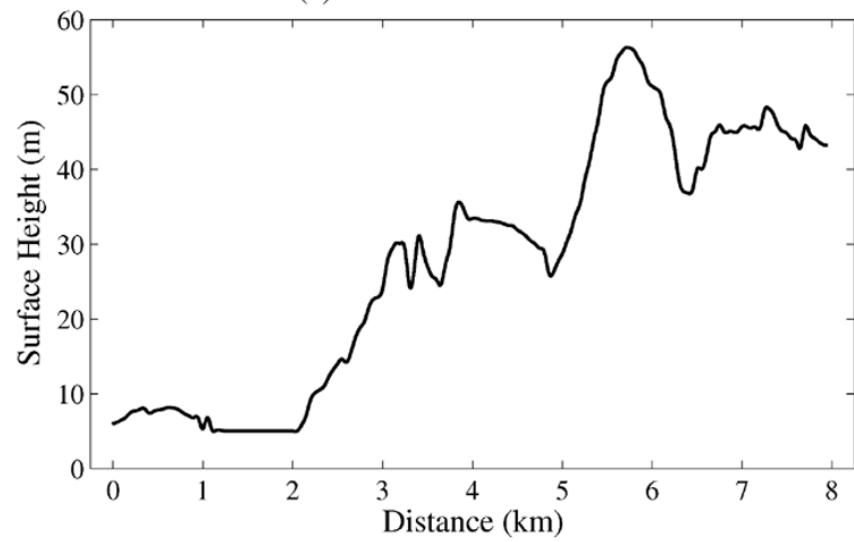

(b) TM Polarization at $435 \mathrm{MHz}$

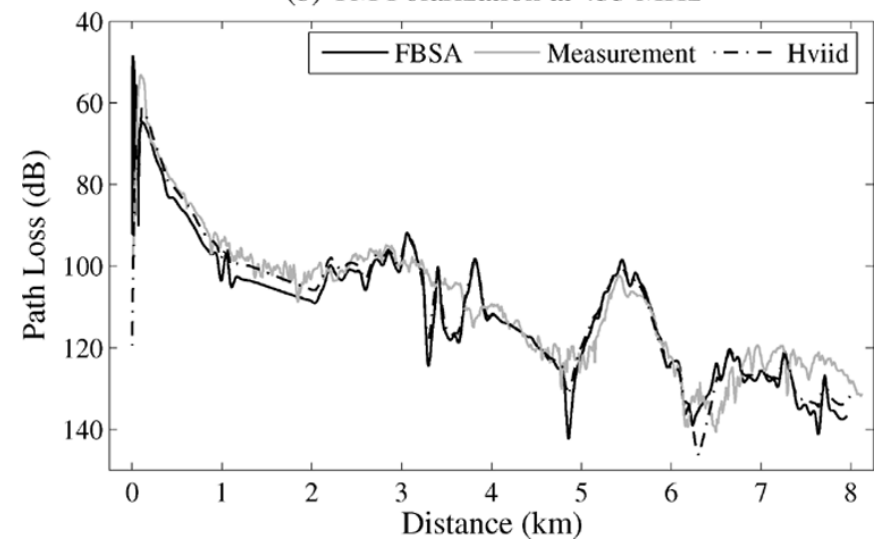

Fig. 2. Path loss over Hadsund terrain profile. (a) Profile geometry. (b) TM polarization at $435 \mathrm{MHz}$. Distance $=\mathbf{7 9 5 0} \mathbf{m}, \boldsymbol{\Delta} \boldsymbol{x}=\mathbf{0 . 1 \lambda}, \boldsymbol{N}=$ $115275, N_{q}=117, L_{s}=13 \lambda$.

Instead of the direct solution of the system defined by (3), which requires $O\left(N^{3}\right)$ operations, the FBSA $(O(N))$ is used in order to find the unknown current coefficients for electrically very large terrains. For further details on the FBSA, the reader is referred to [12] and [13].

\section{NumericAl RESUlts AND Discussion}

\section{A. Validation of the FBSA for Real World Propagation Problems}

In order to assess the accuracy of the FBSA as well as to demonstrate its consistency with measurements, comparisons of FBSA results with measurements are shown in Figs. 2 and 3. The terrain profiles are from Denmark with lengths up to $8 \mathrm{~km}$. The height variations are of the order 20-50 m. Measured data were obtained by Hviid et al. [14] using a dipole with a transmitted power of $10 \mathrm{~W}$ and a gain of $8 \mathrm{dBi}$. The transmitter height is $10.4 \mathrm{~m}$. The receiver antenna is a $\lambda / 4$ monopole on top of a van with a height of $2.4 \mathrm{~m}$. Having no exact information about the vegetation and electrical properties of terrains, the surface impedances are taken as $\eta_{s}=20.2+j 8.1 \Omega$ in order to handle some small forests and other land cover data along the profiles [14]. Also shown in the figures are the computations of Hviid et al. [14] with a different terrain based integral equation method. This method neglects the backscattering, has a computational cost of $O\left(N^{2}\right)$, assumes perfect magnetic conductor terrain, and it can only handle the TM polarization case. We have taken the segment length $\lambda / 10$, and the strong region length, $L_{s}=\left(z_{\max }-z_{\min }\right) / 4$, is calculated as $13 \lambda$ and $6 \lambda$, respectively, for the terrain profiles in Figs. 2(a) and 3(a).

In Fig. 2(b), the results for $435 \mathrm{MHz}$ operating frequency are presented over Hadsund terrain profile, while the comparisons over Jer- (a) Jerslev Terrain Profile

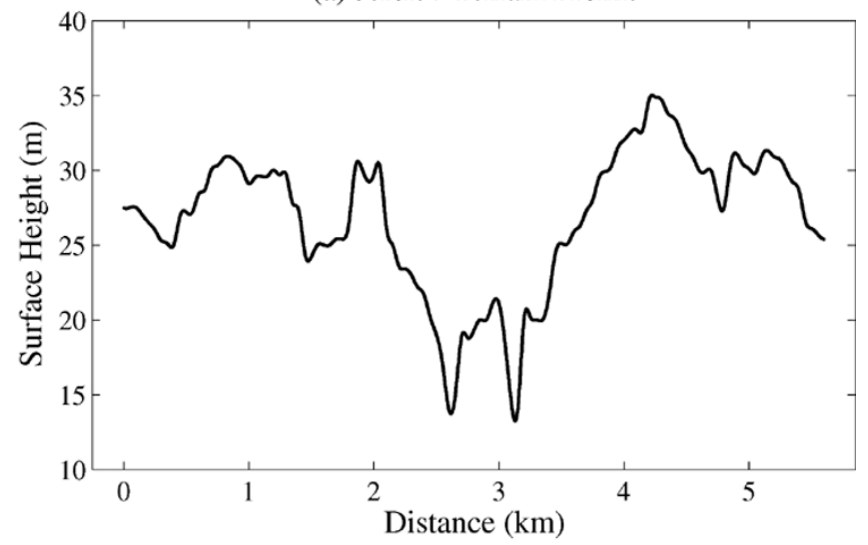

(b) TM Polarization at $970 \mathrm{MHz}$

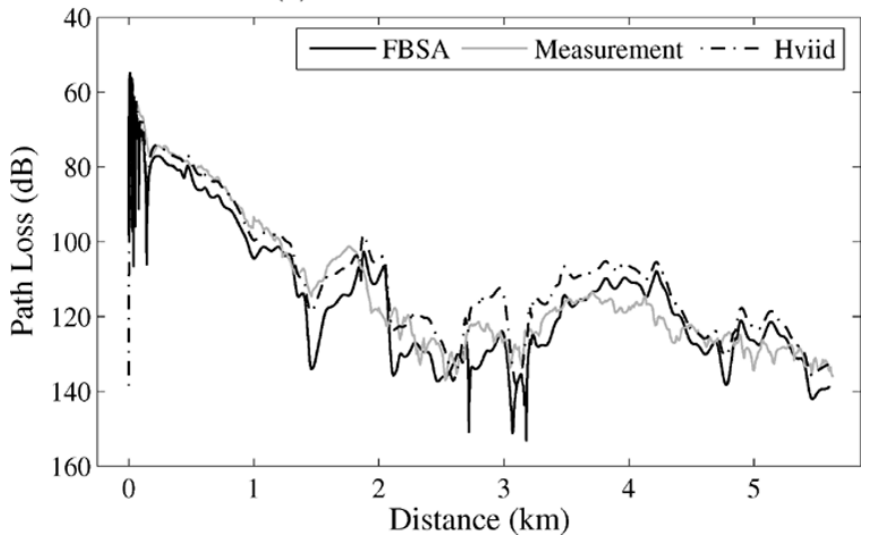

Fig. 3. Path loss over Jerslev terrain profile. (a) Profile geometry. (b) TM polarization at $970 \mathrm{MHz}$. Distance $=\mathbf{5 6 0 0} \mathbf{m}, \boldsymbol{\Delta} \boldsymbol{x}=\mathbf{0 . 1} \boldsymbol{\lambda}, \boldsymbol{N}=$ $185066, N_{q}=124, L_{s}=6 \lambda$.

slev profile for $970 \mathrm{MHz}$ are shown in Fig. 3(b). Both figures show the very good agreement of the FBSA results with the measurements and the other IE method. Therefore, the FBSA can safely be used as a reference solution to test the accuracy of the prediction of various ITU and FCC recommended propagation models.

\section{B. Accuracy of the Prediction of ITU and FCC Propagation Models}

In Fig. 4, the accuracy of three empirical models are compared on an actual terrain profile from Turkey. These models are ITU Recommendation Rec.-529 [2], [3], ITU Recommendation Rec.-1546 [4], and free space propagation model [5] with multiple diffraction. Note that, ITU Rec.-529 is the same as the Hata model [2] at $500 \mathrm{MHz}$. The MD correction used here is due to the Epstein-Peterson [7] in which obstruction from each knife-edge is added consecutively. The surface impedance is taken as a typical value of $\eta_{s}=25+j 20 \Omega$. The transmitter antenna is considered to be an isotropic radiator with a transmitted power of $50 \mathrm{~W}$ and a height of $20 \mathrm{~m}$ located at the left-most end of the terrain. The receiver antenna is taken as an isotropic radiator, too, having a height of $1.8 \mathrm{~m}$. We have taken the strong region length, $L_{s}$, as $5 \mathrm{~m}$ for the terrain profiles in Figs. 4(a) and 5(a).

In Fig. 4(b), the results for the $200 \mathrm{MHz}$ operating frequency are presented for TM polarization. The free space propagation model with MD correction seems to have the best agreement with FBSA. The comparisons for $500 \mathrm{MHz}$ are shown in Fig. 4(c) for TE polarization case. Numerical results show that the best agreement with FBSA results is obtained using free space propagation model with diffraction correction. Also, ITU Rec.-529 results seem to reasonably agree with the FBSA results especially at $500 \mathrm{MHz}$ (Hata), while the poorest agreement is 
(a) Cinarkoy Terrain Profile

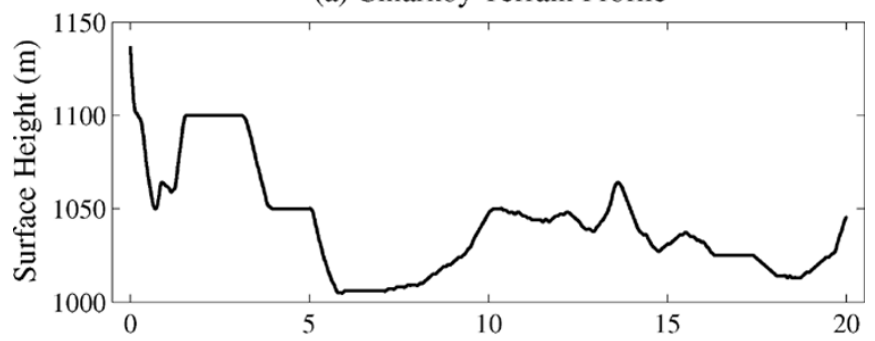

(b) TM Polarization at $200 \mathrm{MHz}$

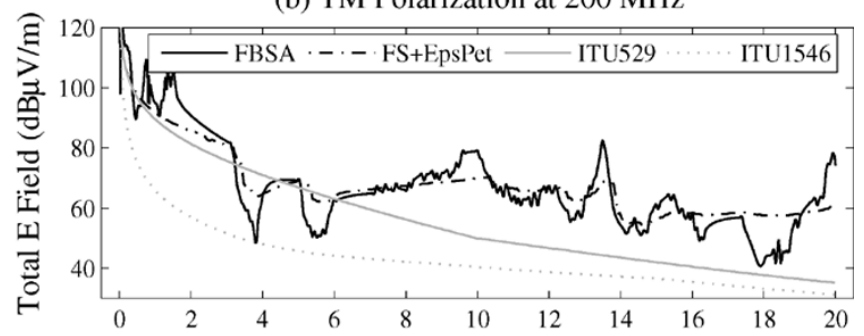

(c) TE Polarization at $500 \mathrm{MHz}$

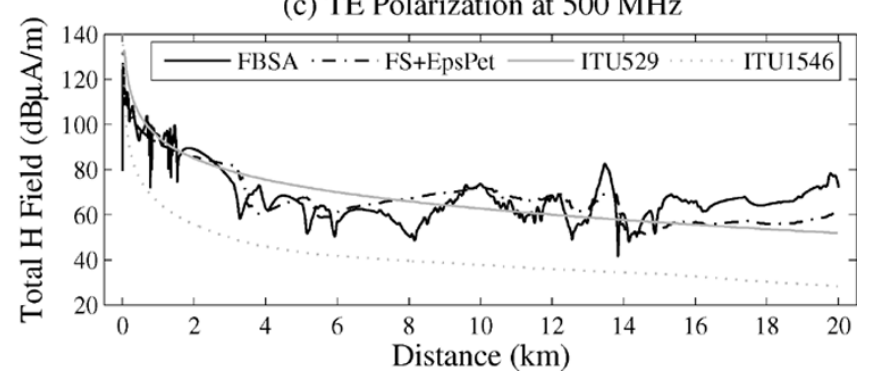

Fig. 4. Total field over Cinarkoy terrain profile. (a) Profile geometry. (b) $\mathrm{TM}$ polarization at $200 \mathrm{MHz}$. Distance $=\mathbf{2 0} \mathbf{~ k m}, \boldsymbol{\Delta} \boldsymbol{x}=\mathbf{0 . 1} \boldsymbol{\lambda}$, $\boldsymbol{N}=133 \mathrm{333}, \boldsymbol{N}_{q}=\mathbf{2 2 5}, \boldsymbol{L}_{s}=\mathbf{5} \mathbf{m}$. (c) TE polarization at $500 \mathrm{MHz}$. Distance $=20 \mathrm{~km}, \Delta x=0.1 \lambda, N=333333, N_{q}=286$, $\boldsymbol{L}_{s}=\mathbf{5} \mathrm{m}$.

obtained for ITU Rec.-1546. We believe that, the use of ITU Rec.-1546 for propagation over rural areas will not be very accurate for ranges up to $20 \mathrm{~km}$. In [4], it is stated that, the ITU Rec.-1546 should yield consistent results with Hata up to $20 \mathrm{~km}$. However, the given Hata equation in the recommendation [4] is the one for urban areas and results in a difference around $25 \mathrm{~dB}$ in path loss from the rural Hata equation at $300 \mathrm{MHz}$ [23]. Therefore, the ITU Rec.-1546 should be modified in order to be used for propagation over terrain profiles in rural areas.

The reason for the choice of the Epstein-Peterson method among the available MD corrections is explained with the aid of Fig. 5(b) and (c) for TM and TE polarizations, respectively. MD loss methods, Bullington [8], Vogler [10] and Epstein-Peterson [7], are examined in conjunction with free space propagation model in these figures. In Bullington method, all knife-edges are replaced by an equivalent one and in Vogler, Fresnel type integrals for each aperture is taken consecutively. Comparisons show that the use of any MD correction model yield quite similar results. However, a careful investigation of the figures show that, the Bullington method cannot catch the diffraction loss effects of some of the consecutive peaks that are close to each other. Vogler method is computationally expensive and yields quite similar results with Epstein-Peterson; still the least deviation from the reference solution occurs with the use of Epstein-Peterson. Therefore, the best choice for the multiple diffraction correction seems to be Epstein-Peterson.

The addition to MD losses to free space propagation, as in Figs. 4 and 5 , predicts the fluctuations in the field strength due to terrain undulations. the same idea can be applied to the empirical propagation models. The effect of using MD losses with empirical propagation models is shown explicitly in Fig. 6. Free space propagation model, ITU Rec.-1546 and (a) Konya Terrain Profile

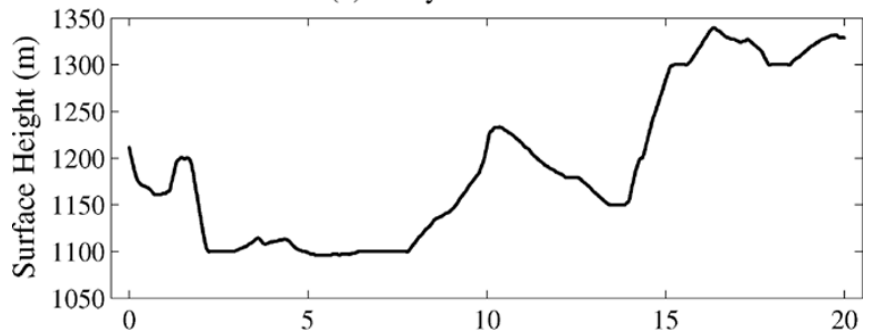

(b) TM Polarization at $200 \mathrm{MHz}$

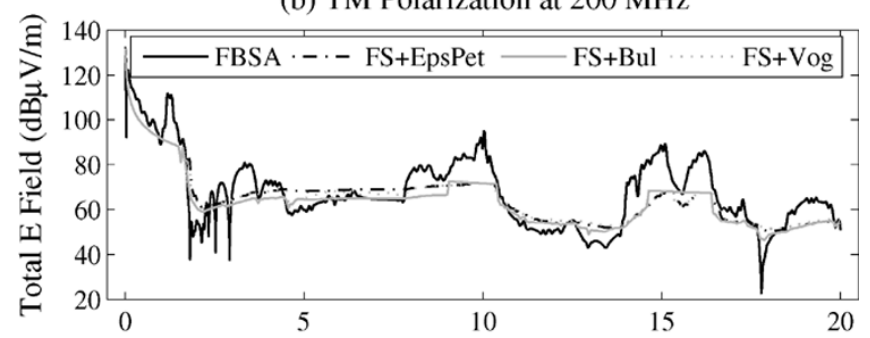

(c) TE Polarization at $200 \mathrm{MHz}$

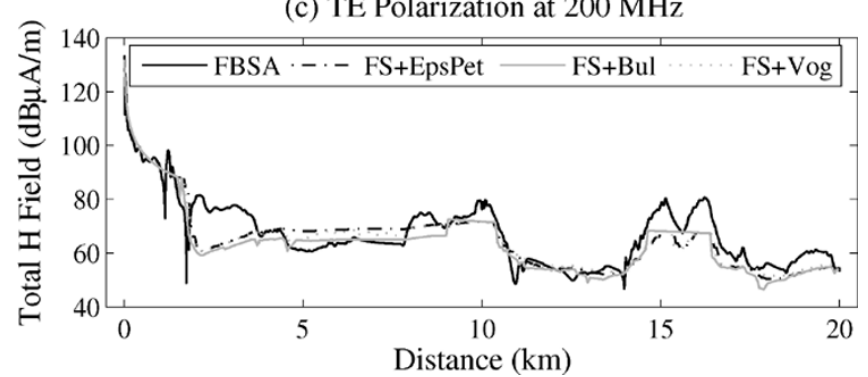

Fig. 5. Comparisons of MDCs over Konya terrain profile. (a) Profile geometry. (b) TM polarization at $200 \mathrm{MHz}$. (c) TE polarization at $200 \mathrm{MHz}$. Distance $=20 \mathrm{~km}, \Delta x=0.1 \lambda, N=133333, N_{q}=286$, $L_{s}=5 \mathrm{~m}$.

FCC curves, with and without MD corrections, are compared in terms of accuracy using the FBSA as a reference, on the same geometry depicted before in Fig. 4(a). The dotted lines in Fig. 6 represent the empirical models only, whereas the solid ones show the models with MD corrections. Reasonable agreement of free space and FCC results with the FBSA solution is observed in conjunction with (and without) MD corrections. In MD corrections, effects of the diffraction phenomenon is taken into account as an additional path loss only. Therefore, when used in conjunction with empirical curves, they just decrease the level of the curve along the portions of the terrain which are out of the lit region of the source. Hence, the use of MD losses together with empirical solutions may increase the error if the empirical curve is at a significantly lower level than the reference solution. Thus, using MD losses with ITU Rec.-1546 increases the error. According to above observations, for the propagation over rural areas, ITU Rec.-1546 deviates much more than the older ITU recommended models.

\section{CONCLUSION}

Most widely used empirical propagation models with MD corrections for prediction of the field strengths over large terrain profiles have been investigated and observed that, they cannot provide a very accurate estimation of the scattered field or the path loss for an arbitrary environment, since they are polarization independent and do not respond to changes in electrical properties of the terrain.

Furthermore, special care is needed when MD correction methods are used in conjunction with empirical solutions. Implementation of an MD correction method increases the error if the field values due to an empirical model is already lower than the reference solution. 
TM Polarization at $200 \mathrm{MHz}$

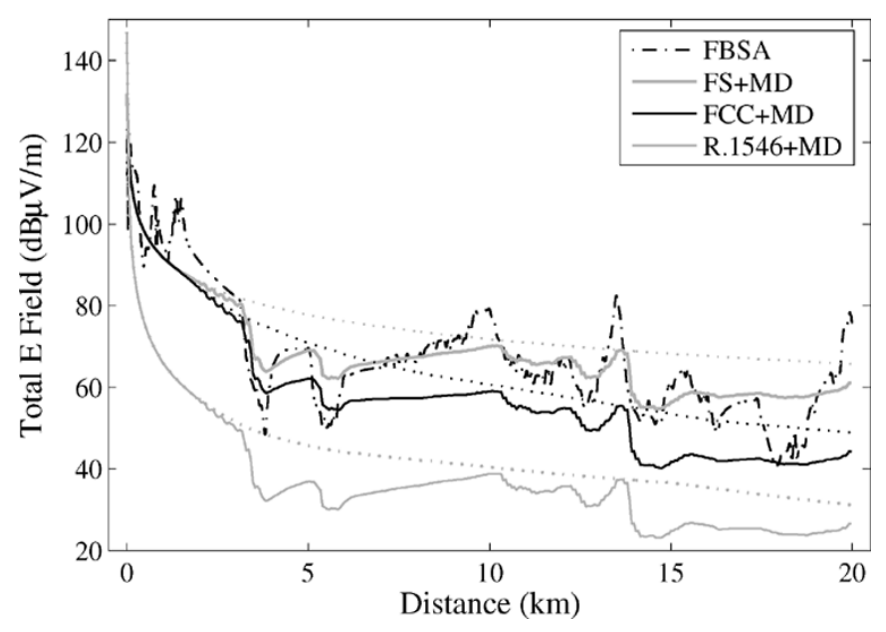

Fig. 6. Comparisons of propagation models with and without MD corrections over Cinarkoy terrain profile for TM polarization at $200 \mathrm{MHz}$. Distance = $20 \mathrm{~km}, \Delta x=0.1 \lambda, N=133333, N_{q}=225, L_{s}=5 \mathrm{~m}$.

An interesting result of this study is the accuracy of the Rec.-1546, which is one of the most recent ITU recommended propagation models. For urban areas, it is consistent with Hata equations up to about $20 \mathrm{~km}$, but for rural areas, predicted field values of this model deviate from the reference solution more than those of the older ITU models do. Therefore it needs to be modified for rural areas.

The results of this study may help in the choice of the most preferable empirical models or in the development of more robust propagation techniques. A robust technique for the prediction of field strengths over large terrain profiles must be polarization and frequency dependent, and must take electrical properties, shadow and lit regions of the terrain profile into account.

\section{ACKNOWLEDGMENT}

The authors thank Prof. J. B. Andersen (Aalborg University, Denmark) and his associates for kindly providing the terrain profiles and integral equation method results. They also thank Dr. S. Topcu of Bilkent University Communications and Spectrum Management Research Center (ISYAM) for supplying implementations of empirical propagation models and multiple diffraction corrections.

\section{REFERENCES}

[1] Y. Okumura, E. Ohmori, T. Kawano, and K. Fukuda, "Field strength and its variability in vhf and uhf land-mobile radio service," Rev. Elect. Commun. Lab., vol. 16, pp. 825-873, Sep. 1968.

[2] M. Hata, "Empirical formula for propagation loss in land mobile radio services," IEEE Trans. Veh. Technol., vol. 29, pp. 569-571, Aug. 1980.

[3] "Prediction Methods for the Terrestrial Land Mobile Service in the VHF and UHF Bands," International Telecommunication Union, Recommendation ITU-R P.529-3, 1999.

[4] "Method for Point-to-Area Predictions for Terrestrial Services in the Frequency Range $30 \mathrm{MHz}$ to $3000 \mathrm{MHz}$," International Telecommunication Union, Recommendation ITU-R P.1546, 2001.

[5] "Calculation of Free-Space Attenuation," International Telecommunication Union, Recommendation ITU-R PN.525-2, 1994.

[6] "Propagation by Diffraction," International Telecommunication Union, Recommendation ITU-R PN.526-7, 2001.

[7] J. Epstein and D. Peterson, "An experimental study of wave propagation at $850 \mathrm{mhz}$," Proc. IEEE, vol. 41, pp. 595-611, 1953.

[8] K. Bullington, "Radio propagation at frequencies above 30 megacycles," Proc. IRE, vol. 35, pp. 1122-1136, Oct. 1947.
[9] J. Deygout, "Multiple knife-edge diffraction of microwaves," IEEE Trans. Antennas Propag., vol. AP-14, no. 4, pp. 480-489, Jul. 1966.

[10] L. E. Vogler, "An attenuation function for multiple knife-edge diffraction," Radio Sci., vol. 17, pp. 1541-1546, Nov. 1982.

[11] D. Holliday, L. L. D. Jr., and G. J. St.-Cyr, "Forward-backward: A new method for computing low-grazing scattering," IEEE Trans. Antennas Propag., vol. 44, no. 5, pp. 722-729, May 1996.

[12] H.-T. Chou and J. T. Johnson, "A novel acceleration for the computation of scattering from rough surfaces with the forward-backward method," Radio Sci., vol. 33, pp. 1277-1287, Jun. 1998.

[13] J. A. López, M. R. Pino, F. Obelleiro, and J. L. Rodríguez, "Application of the spectral acceleration forward-backward method to coverage analysis over terrain profiles," J. Electromagn. Waves Appl., vol. 15, pp. 1049-1074, Aug. 2001.

[14] J. T. Hviid, J. B. Anderson, J. Toftgard, and J. Bojer, "Terrain based propagation model for rural area - An integral equations approach," IEEE Trans. Antennas Propag., vol. 43, no. 1, pp. 41-46, Jan. 1995.

[15] R. Janaswamy, "A fredholm integral equation method for propagation over small terrain irregularities," IEEE Trans. Antennas Propag., vol. 40, no. 9, pp. 1416-1422, Sep. 1994.

[16] J. T. Johnson, R. T. Shin, J. C. Eidson, L. Tsang, and J. A. Kong, "A method of moments model for vhf propagation," IEEE Trans. Antennas Propag., vol. 45, no. 1, pp. 115-125, Jan. 1997.

[17] C. Brennan and P. J. Cullen, "Application of the fast far-field approximation to the computation of uhf pathloss over irregular terrain," IEEE Trans. Antennas Propag., vol. 46, no. 6, pp. 881-890, Jun. 1998.

[18] — , "Tabulated interaction method for uhf terrain propagation problems," IEEE Trans. Antennas Propag., vol. 46, no. 5, pp. 738-739, May 1998.

[19] C. L. Rino and H. D. Ngo, "Forward propagation in a half-space with an irregular boundary," IEEE Trans. Antennas Propag., vol. 45, no. 9, pp. 1340-1347, Sep. 1997.

[20] C. L. Rino and V. R. Kruger, "A comparison of forward-boundary-integral and parabolic-wave-equation propagation models," IEEE Trans. Antennas Propag., vol. 49, no. 4, pp. 574-582, Apr. 2001.

[21] F. K. Akorli and E. Costa, "An efficient solution of an integral equation applicable to simulation of propagation along irregular terrain," IEEE Trans. Antennas Propag., vol. 49, no. 7, pp. 1033-1036, Jul. 2001.

[22] T. B. A. Senior, "Impedance boundary conditions for imperfectly conducting surfaces," Appl. Sci. Res., vol. 8, pp. 418-436, 1961.

[23] T. S. Rappaport, Wireless Communications: Principles and Practice, 2nd ed. Englewood Cliffs, NJ: Prentice Hall, 2001.

\section{A Low Profile Single Dipole Antenna Radiating Circularly Polarized Waves}

Fan Yang and Yahya Rahmat-Samii

\begin{abstract}
A low profile single dipole antenna that can generate circularly polarized $(\mathrm{CP})$ radiation patterns is proposed in this paper. The CP patterns and low profile configuration are achieved using a specially designed artificial ground plane: a thin grounded slab loaded with periodic rectangular patches. The artificial ground plane exhibits in-phase reflection coefficients with polarization-dependent feature. The radiation mechanism of the antenna is described, and experimental results verify the antenna concept.
\end{abstract}

Index Terms-Artificial ground plane, circular polarization, dipole antenna, low profile.

Manuscript received January 22, 2004; revised December 1, 2004.

F. Yang was with the Electrical Engineering Department, University of California, Los Angeles, CA 90095 USA. He is now with the Electrical Engineering Department, University of Mississippi, University, MS 38677 USA (e-mail: fyang@olemiss.edu).

Y. Rahmat-Samii is with the Electrical Engineering Department, University of California, Los Angeles, CA 90095 (e-mail: rahmat@ee.ucla.edu).

Digital Object Identifier 10.1109/TAP.2005.854536 